\title{
Actualidades Pedagógicas
}

2019-01-01

\section{Las aventuras de Huckleberry Finn: una herramienta de la literatura para desarrollar las habilidades del inglés como lengua extranjera y para entender la cultura norteamericana del siglo XIX}

Yeison Mauricio Cárdenas Reyes

Universidad de La Salle, Bogotá, yecardenas@unisalle.edu.co

Follow this and additional works at: https://ciencia.lasalle.edu.co/ap

\section{Citación recomendada}

Cárdenas Reyes, Y. M.. (2019). Las aventuras de Huckleberry Finn: una herramienta de la literatura para desarrollar las habilidades del inglés como lengua extranjera y para entender la cultura norteamericana del siglo XIX. Actualidades Pedagógicas, (73), 97-117. doi:https://doi.org/10.19052/ap.vol1.iss73.6

This Artículo de Investigación is brought to you for free and open access by the Revistas científicas at Ciencia Unisalle. It has been accepted for inclusion in Actualidades Pedagógicas by an authorized editor of Ciencia Unisalle. For more information, please contact ciencia@lasalle.edu.co. 


\title{
Las aventuras de Huckleberry Finn: una herramienta de la literatura para desarrollar las habilidades del inglés como lengua extranjera y para entender la cultura norteamericana del siglo XIX
}

\author{
Yeison Mauricio Cárdenas Reyes \\ Universidad de La Salle, Bogotá, Colombia \\ yecardenas@unisalle.edu.co
}

Resumen: Este artículo discute y propone un conjunto de enfoques y propuestas didácticas para la enseñanza del inglés como lengua extranjera y de la cultura norteamericana, por medio de la obra literaria Las aventuras de Huckleberry Finn, escrita por Mark Twain, a los estudiantes de licenciatura en lenguas extranjeras. Este artículo aborda tres grandes ejes: 1) la relevancia didáctica y discursiva del texto para su respectivo estudio en el aula de clase; 2) la instrumentalización del libro para entender el contexto sociocultural de la época, y 3) la reflexión sobre cómo didácticamente se puede establecer un dispositivo pedagógico que permita el análisis de dos temas principales del libro, como lo son la religión y la esclavitud.

Palabras clave: literatura, enseñanza, inglés, lengua, competencias, didáctica, cultura.

Recibido: 13 de junio de 2018 Aceptado: 10 de septiembre de 2018

Cómo citar este artículo: Cárdenas Reyes, Y. M. (2019). Las aventuras de Huckleberry Finn: una herramienta de la literatura para desarrollar las habilidades del inglés como lengua extranjera y para entender la cultura norteamericana del siglo XIX. Actualidades Pedagógicas, (73), 97-117. https://doi.org/10.19052/ap.vol1. iss 73.5 


\section{Adventures of Huckleberry \\ Finn: A Literary Tool to Develop \\ Skills in English as a Foreign \\ Language and to Understand \\ North American Culture during \\ the Nineteenth-Century}

Abstract: This paper discusses and proposes a set of didactic approaches and proposals for teaching English as a foreign language and North American culture through Mark Twain's Adventures of Huckleberry Finn to students from the Bachelor's Degree in Foreign Languages. The paper addresses three main topics: 1) the didactic and discursive relevance of the text for their respective study in the classroom; 2) the instrumentalization of the book to understand the sociocultural context of the time; and 3) the reflection on how to didactically establish a pedagogical device to analyze two main themes of the book, such as religion and slavery.

Keywords: literature, teaching, English, language, competences, didactics, culture.

\section{As aventuras de Huckleberry Finn: uma ferramenta de literatura para desenvolver as habilidades do inglês como língua estrangeira e entender a cultura norte-americana do século XIX}

Resumo: Este artigo discute e propõe um conjunto de abordagens e propostas didáticas para o ensino do inglês como língua estrangeira e da cultura norte-americana através da obra literária As aventuras de Huckleberry Finn, escrita por Mark Twain aos estudantes de graduação em línguas estrangeiras. Este artigo aborda três eixos principais: 1) a relevância didática e discursiva do texto para o respectivo estudo na sala de aula; 2) a instrumentalização do livro para compreender o contexto sociocultural da época, e 3) a reflexão sobre como é possível estabelecer didaticamente um dispositivo pedagógico que permita a análise de dois temas principais do livro como religião e escravidão.

Palavras chave: literatura, ensino, inglês, língua, competências, didática, cultura. 


\section{Introducción}

El entendimiento de una cultura puede definirse por las experiencias de vida de un individuo o el contacto diario de este con la sociedad a la que pertenezca. Se podría aseverar que las ciencias sociales se encargan de estudiar los modos de vida y las costumbres (cultura). Igualmente, resulta interesante consultar las ciencias del lenguaje, ya que estas son las que mejor permiten plasmar conductas y pensamientos en un idioma dado. La escritura, por ejemplo, es un proceso mental, en el que se retratan acciones o eventos según la persona que los relate. De acuerdo con autores como Flower y Hayes (1981) y Wang y Wen (2002, citados en Villalobos, 2017), esta habilidad del lenguaje es "una de las actividades mentales más complejas y, por lo tanto, una de las más difíciles de desarrollar" (p. 46). Ahora bien, la literatura entraría a ser un elemento clave en el propósito de transmitir y conformar saberes en la idiosincrasia de una sociedad. Si se abordara un análisis epistemológico de la palabra "literatura", se observaría que viene del latín litterae, que significa "acumulación de saberes para escribir y leer de modo correcto" (Pérez Porto y Merino, 2012); esto significa que gracias a la literatura se puede ilustrar corrientes de pensamiento a través de símbolos y convenciones escritas, al igual que relatar hechos históricos y manifestaciones culturales de las sociedades. De esta manera, se podrían considerar estos escritos como artísticos, si se tiene en cuenta la definición de la Real Academia Española (2018) con respecto a la literatura: "Arte de la expresión verbal". Este arte no solamente alimentaría el hambre de conocimiento, sino que establecería las bases para saber interpretar las diferentes culturas del mundo y las sociedades que las conforman. Dado lo anterior, la obra Las aventuras de Huckleberry Finn (escrita en 1885), del estadounidense Mark Twain (1835-1910), que es un texto literario reconocido en el mundo tanto en el campo literario como en los ámbitos cultural e histórico, merece un análisis desde tres perspectivas: la primera es su injerencia didáctica en el aprendizaje de los estudiantes de licenciatura en inglés y lenguas extranjeras; la segunda es analizar cómo el libro se convierte en un instrumento 
literario para entender la cultura y la sociedad norteamericana del siglo XIX, y la tercera es una reflexión o apreciación didáctica de los elementos culturales transmitidos por el texto de Twain.

\section{Injerencia didáctica de Las aventuras de Huckleberry Finn en el aprendizaje de los estudiantes de licenciatura en inglés y lenguas extranjeras}

En principio, la literatura reglamenta ciertas conductas necesarias para la mejora de las competencias del lenguaje. El Marco común europeo de referencia para las lenguas (Consejo de Europa, 2002) resalta estos procedimientos como "procesos", los cuales define: "Los procesos se refieren a la cadena de acontecimientos, neurológicos y fisiológicos, implicados en la expresión y en la comprensión oral y escrita” (p. 24). Esto se traduce en una serie de conexiones cerebrales y operaciones del cuerpo humano que conllevan al fortalecimiento de la competencia en recepción escrita; además, se establece una estrecha relación entre la lectura y las prácticas sociales, como lo resalta Piaget (1979, citado en Papo y Vourgain, 1989): "La liaison constitutive de toute connaissance [...] est l'assimilation des objets à des schémas” (p. 33). ${ }^{1}$

En otras palabras, interacciones comunicativas, como leer para informarse, serían clave para movilizar un conjunto de componentes de tipo sintáctico, semántico, lexical, fonológico y pragmático, para llevar a cabo tal savoir-faire en una lengua extranjera, como el inglés como lengua extranjera (ILE). Ahora, el texto de Twain (1885) es la herramienta literaria ideal para poner en marcha un dispositivo didáctico que entrelace las competencias lingüísticas con la competencia enciclopédica, las cuales permitan identificar el contexto del autor y la obra. Debido a esto, se podría proponer un esquema que ilustre tal dispositivo didáctico en el aula de ILE o lenguas extranjeras en general (figura 1).

1 "El vínculo constitutivo de todo conocimiento [...] es la asimilación de los objetos a patrones". 
Figura 1. Diferencia entre el eje del tratamiento didáctico y la relación pedagógica

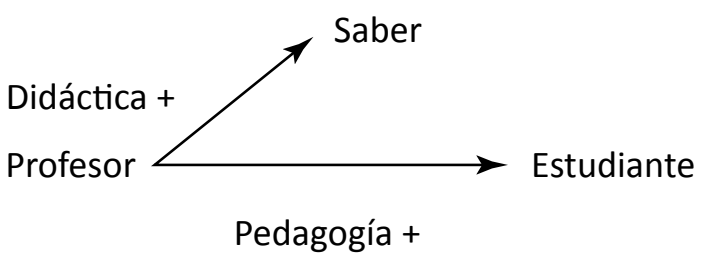

Fuente: tomada y traducida del francés de Descotes, Jordy y Langlade (1993, p. 109).

El papel del docente es crear un escenario académico en el cual pueda transmitir un saber; para lograrlo, necesita de elementos didácticos propios de la enseñanza de la literatura y de las competencias en recepción escrita. Al respecto, existen varias estrategias que son comunes para tener mejores hábitos de lectura; entre estas, podemos resaltar: skimming, scanning, resúmenes, mapas mentales y crear una lista de palabras clave. El saber puede estimarse según el objetivo comunicativo que quiera lograrse; así, una vez esté claro el vector transmisión de saberes, se plantea la forma de hacer al estudiante partícipe en la apropiación de estos.

La obra de Twain es una novela característica de la literatura moderna del siglo XIX, la cual tiene lugar alrededor del río Mississippi. Su personaje principal es Huckleberry Finn, quien a su vez es el narrador de la historia. El libro posee rasgos culturales y étnicos particulares, como los dialectos y las expresiones afroamericanos y de los señores feudales, quienes eran los blancos. Su tipología textual es la narrativa. Así mismo, se caracteriza por marcar grandes eventos socioculturales de la época, como la esclavitud, la influencia de la tradición cristiana en su población y los conflictos entre familias en los Estados Unidos.

Esta obra literaria es un texto pertinente para los estudiantes que estén en una licenciatura en lenguas extranjeras, en especial ILE, y para quienes se encuentren culminando su nivel A2+ y comenzando el B1. Ya que hacen uso de ciertos componentes lingüísticos propios de tal escala de nivel de competencia del Marco común europeo de referencia para las lenguas, cuando se contrasta sus formas lingüísticas, se plantea el referencial de programas de la Alianza Francesa (Chauvet, 2008) para tal fin. 
De esta manera, se tomarán fragmentos de Las aventuras de Huckleberry Finn, escrita en su idioma original (inglés), con el fin de señalar con ejemplos específicos por qué la obra es pertinente para trabajar desde el nivel A2+ y pasar al B1 desde lo gramatical, lexical y pragmático (tablas 1, 2 y 3 ).

Tabla 1. Competencias lingüísticas

\begin{tabular}{|c|c|c|}
\hline Fragmento & Competencia gramatical & $\begin{array}{c}\text { Nivel de } \\
\text { competencia }\end{array}$ \\
\hline $\begin{array}{l}\text { "Next, I got a large bag and filled it } \\
\text { with rocks" (p. 21). }\end{array}$ & $\begin{array}{l}\text { Uso del pasado simple (Chauvet, } \\
2008, \text { p. 48). }\end{array}$ & $\mathrm{A} 2$ \\
\hline $\begin{array}{l}\text { "No, forget the candles. Jim'll wake } \\
\text { up, and then the Widow will learn } \\
\text { that I'm not in bed" (p. 4). }\end{array}$ & $\begin{array}{l}\text { Uso del futuro simple con auxiliar } \\
\text { will (Chauvet, 2008, p. 48). }\end{array}$ & $\mathrm{A} 2$ \\
\hline \multirow{2}{*}{$\begin{array}{l}\text { "While I was lighting a candle, } \\
\text { Jim lay down on his blanket and } \\
\text { suddenly screamed" (p. 4). }\end{array}$} & $\begin{array}{l}\text { Uso del pasado simple y continúo } \\
\text { para realizar narraciones. }\end{array}$ & \multirow[b]{2}{*}{$\mathrm{A} 2$} \\
\hline & $\begin{array}{l}\text { Uso de adverbios temporales para } \\
\text { ubicar una acción en una idea: } \\
\text { while (Chauvet, 2008, p. 48). }\end{array}$ & \\
\hline $\begin{array}{l}\text { "What happens if the boat } \\
\text { doesn't sink?" (p. 41). }\end{array}$ & $\begin{array}{l}\text { El condicional en presente para la } \\
\text { duda (Chauvet, 2008, p. 101). }\end{array}$ & B1 \\
\hline $\begin{array}{l}\text { "Finally, he was too tired to } \\
\text { move. He took a blanket and } \\
\text { wrapped it around himself and } \\
\text { lay on the floor. He would cry } \\
\text { quietly, then shout, then would cry } \\
\text { again" (p. 18). }\end{array}$ & $\begin{array}{l}\text { Hechos anteriores, simultáneos } \\
\text { y posteriores: concordancia en } \\
\text { tiempo (Chauvet, 2008, p. 101). }\end{array}$ & B1 \\
\hline
\end{tabular}

Tabla 2. Competencia lexical

\begin{tabular}{|l|r|}
\hline \multicolumn{1}{|c|}{ Lista de vocabulario } & \multicolumn{1}{|c|}{$\begin{array}{c}\text { Nivel de } \\
\text { competencia }\end{array}$} \\
\hline $\begin{array}{l}\text { Los lugares } \\
\text { La vida cotidiana: el trabajo, las actividades cotidianas. }\end{array}$ & A2 \\
\hline $\begin{array}{l}\text { Política } \\
\text { La religión } \\
\text { La educación } \\
\text { La cultura } \\
\text { La historia } \\
\text { La salud } \\
\text { Las problemáticas sociales (Chauvet, 2008, p. 98) }\end{array}$ \\
\hline
\end{tabular}


Tabla 3. Competencia pragmática

\begin{tabular}{|c|c|c|c|}
\hline Fragmento del libro & $\begin{array}{c}\text { Competencia } \\
\text { pragmática }\end{array}$ & \multicolumn{2}{|c|}{ Nivel de competencia } \\
\hline $\begin{array}{l}\text { "Some said that he died } \\
\text { in prison, but others } \\
\text { said that he escaped to } \\
\text { America" (p. 47). }\end{array}$ & $\begin{array}{l}\text { Uso de conector lógicos } \\
\text { simples: but, because } \\
\text { (Chauvet, 2008, p. 63). }\end{array}$ & \multicolumn{2}{|c|}{$\mathrm{A} 2$} \\
\hline Fragmento del libro & $\begin{array}{l}\text { Competencia } \\
\text { pragmática }\end{array}$ & Tipo de conector & $\begin{array}{c}\text { Nivel de } \\
\text { competencia }\end{array}$ \\
\hline $\begin{array}{l}\text { "[...] therefore, I simply } \\
\text { allowed the current to } \\
\text { carry me downstream. } \\
\text { (p. 49). }\end{array}$ & $\begin{array}{l}\text { Uso de conectores lógi- } \\
\text { cos para expresar causa, } \\
\text { consecuencia y oposición } \\
\text { (Chauvet, 2008, p. 90). }\end{array}$ & Consecuencia & B1 \\
\hline $\begin{array}{l}\text { "However, he said that } \\
\text { it often made him feel } \\
\text { better if people treated } \\
\text { him like a King" (p. 71). }\end{array}$ & $\begin{array}{l}\text { Uso de conectores lógi- } \\
\text { cos para expresar causa, } \\
\text { consecuencia y oposición } \\
\text { (Chauvet, 2008, p. 90). }\end{array}$ & Oposición & B1 \\
\hline
\end{tabular}

Igualmente, es necesario considerar:

[...] a partir de los textos literarios, la competencia literaria activa los distintos conocimientos para reconocer los usos y convenciones comunicativas de la lengua. De modo que el aprendiz — que interviene como lector-genera las actividades de aprendizaje a través de la lectura. (Mendoza, 2008)

El mismo Mendoza (2008) esquematiza su perspectiva (figura 2). De esta manera, se reafirma que la obra de Twain (1885) puede implementarse en la clase de inglés para terminar el nivel A2+ e introducir el B1, porque la estructuración de sus contenidos es acorde con las necesidades de aprendizaje y comunicativas de los estudiantes. Por lo tanto, es necesario poner en funcionamiento un dispositivo que asegure el aprendizaje de todas las competencias de la lengua. Este artículo aboga, consecuentemente, por un enfoque transversal que integre competencias, saberes y didácticas, las cuales, posteriormente, serán desarrolladas y adquiridas en función de un savoir-faire comunicativo por el estudiante de licenciatura en ILE o de lenguas extranjeras en el aula de clase. En la figura 3 se esquematiza este propósito, el cual permite la transmisión de conocimientos y saberes al estudiante, que se deriven de la obra de Twain. 
Figura 2. La competencia literaria y la competencia comunicativa

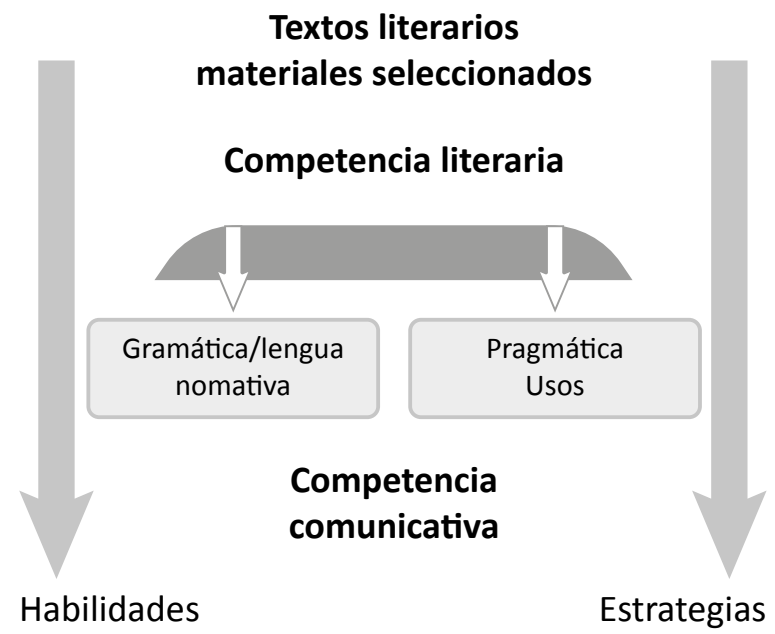

Fuente: Mendoza (2008).

Figura 3. Hacer del francés en el liceo

\section{Hacer del francés en el liceo}

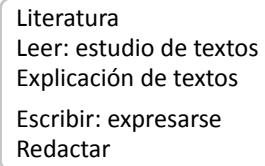

Saber: historia, instrucción
Vocabulario: significaciones de palabras, expresarse, lenguaje, diccionario.

\section{Lenguaje-comunicar: hablar, discutir con los demás, escuchar, expresarse, argumentar, debatir.}

Trabajo: aprender las reglas (gramática), cinco horas/semana, tarea, dificultad, estudiar.
Educarse: ambición

Espíritu: pensar, reflexión, análisis, concentrarse, argumentar, imaginar, discernir, racionamiento

Fuente: tomada y traducida del francés de Descotes, Jordy y Langlade (1993, p. 21 ).

Como se puede evidenciar en la figura 3, hay un conjunto de elementos de toda índole - -lingüísticos, didácticos, pedagógicos, psicológicos y cognitivos-, los cuales se entrelazan en función de un objetivo comunicativo. Por ende, en la implementación de un trabajo literario en el aula de ILE y lenguas, el docente es el encargado de construir un dispositivo didáctico y 
pedagógico que suscite el desarrollo secuencial, armónico y transversal de las competencias y los saberes que presenta el esquema. Este es un ejemplo de secuencia, durante la lectura de un capítulo de la obra de Twain (1885), que se podría llevar a cabo con el estudiante:

- Pedir al estudiante que investigue acerca los hechos que ocurrieron en la época en la que el autor escribió el libro; preparar notas acerca del contexto sociocultural de la época y la vida del autor.

- Realizar lecturas dirigidas; discutir eventos específicos del libro; analizar el contexto, la obra y el autor con base en fragmentos del libro.

- Poseer un cuadernillo con el vocabulario desconocido.

- Hacer ejercicios tipo quiz, en los que se examine el significado de ciertas palabras que aparezcan en el texto.

- Realizar ejercicios gramaticales a partir de ejemplos tomados de fragmentos del libro.

- Finalmente, redactar textos escritos de tipo argumentativo y analítico, para tomar una posición con respecto a un tema que sobresalga en el libro. En este caso, se recomendaría escribir acerca de la esclavitud o la religión en el siglo XIX en Estados Unidos.

Si se ponen en marcha estas recomendaciones, se supone que el estudiante vería cómo su aprendizaje se materializa; al fin y al cabo, lo que debería primar es comunicar en inglés lengua extranjera. De este modo, de acuerdo con Mckay (2001, citado en Gómez, 2015):

\footnotetext{
La literatura les demuestra a los estudiantes de inglés la importancia de lograr metas comunicativas específicas, al igual que proporciona bases para el desarrollo de las cuatro habilidades de la lengua, pues a partir del texto literario los estudiantes pueden leer, hablar, escuchar y escribir sobre los temas, los conflictos y las acciones de los personajes que aparecen en las narraciones. (p. 87)
}

Para terminar, varios autores afirman que emplear la literatura en el aula de lenguas y culturas extranjeras contribuye a desarrollar la calidad en la experiencia de aprendizaje y enseñanza, al igual que la sensibilidad de la persona con respecto a la sociedad que se ilustra en el texto. En la tabla 4 se exponen cuáles son sus puntos en común. 
Tabla 4. Aportes literarios para la enseñanza de lenguas extranjeras y la cultura de sus hablantes

\begin{tabular}{|c|c|c|}
\hline Autor & Citación & $\begin{array}{l}\text { Aporte de la } \\
\text { literatura }\end{array}$ \\
\hline $\begin{array}{l}\text { Federación de } \\
\text { Enseñanza } \\
\text { de Comisiones } \\
\text { Obreras de } \\
\text { Andalucía (2011) }\end{array}$ & $\begin{array}{l}\text { "Se trata de material autentico. Cuando trabaja- } \\
\text { mos en clase con un texto literario le brindamos a } \\
\text { nuestro alumnado la oportunidad de aprender el } \\
\text { idioma tan cual, algo que los libros de textos nos } \\
\text { les ofrecen" (p. 3). }\end{array}$ & $\begin{array}{l}\text { Ofrece un uso del } \\
\text { lenguaje auténtico. }\end{array}$ \\
\hline Mendoza (2008) & $\begin{array}{l}\text { "Los textos literarios sirvieron de material y } \\
\text { de modelo para conducir aprendizajes de cariz } \\
\text { gramatical o para el estudio, en abstracto, de un } \\
\text { modelo de lengua". }\end{array}$ & $\begin{array}{l}\text { Ofrece un uso del } \\
\text { lenguaje auténtico. }\end{array}$ \\
\hline $\begin{array}{l}\text { Kramsch (2001, } \\
\text { citado en Gómez, } \\
\text { 2015) }\end{array}$ & $\begin{array}{l}\text { "[...] el texto literario es lenguaje auténtico y } \\
\text { puede tender a lo que Kramsch (2001) sugiere: } \\
\text { la necesidad de exponer a los estudiantes de } \\
\text { ILE a distintos tipos de materiales auténticos, } \\
\text { incluyendo artículos de revista, periódicos y } \\
\text { lenguaje narrativo y literario, porque ofrecen } \\
\text { oportunidades para la construcción genuina de } \\
\text { significado" (p. 88). }\end{array}$ & $\begin{array}{l}\text { Ofrece un uso del } \\
\text { lenguaje auténtico. }\end{array}$ \\
\hline $\begin{array}{l}\text { Federación de } \\
\text { enseñanza } \\
\text { de Comisiones } \\
\text { Obreras de } \\
\text { Andalucía (2011) }\end{array}$ & $\begin{array}{l}\text { "Herramienta de acercamiento cultural. Los textos } \\
\text { literarios son fuente de cultura, historia y política } \\
\text { de un pueblo". } \\
\text { "Los textos literarios les servirán de guía para } \\
\text { comprender la nueva cultura a la que se enfrenta } \\
\text { al estudiar un idioma, con sus creencias, valores y } \\
\text { necesidades". }\end{array}$ & $\begin{array}{l}\text { Herramienta } \\
\text { para entender la } \\
\text { cultura en la que se } \\
\text { enmarca el libro. }\end{array}$ \\
\hline Mendoza (2008) & $\begin{array}{l}\text { "La competencia comunicativa del alumno } \\
\text { permite el intercambio oral y escrito y, además, } \\
\text { también interviene en la comprensión lectora y } \\
\text { conduce al aprendiz hacia la participación en las } \\
\text { diversas manifestaciones culturales de la comuni- } \\
\text { dad propia de la lengua meta". }\end{array}$ & $\begin{array}{l}\text { Herramienta } \\
\text { para entender la } \\
\text { cultura en la que se } \\
\text { enmarca el libro. }\end{array}$ \\
\hline $\begin{array}{l}\text { Zafeiriadou (2001, } \\
\text { citado en Gómez, } \\
\text { 2015) }\end{array}$ & $\begin{array}{l}\text { “[...] la literatura posibilita la compresión y apre- } \\
\text { ciación de culturas e ideologías diferentes a las } \\
\text { propias, porque esta preserva el legado artístico } \\
\text { y cultural del sentido humano de un grupo en } \\
\text { particular" (p. 88). }\end{array}$ & $\begin{array}{l}\text { Herramienta } \\
\text { para entender la } \\
\text { cultura en la que se } \\
\text { enmarca el libro. }\end{array}$ \\
\hline
\end{tabular}

Fuente: elaboración propia.

Estos aportes que hace la literatura reflejan, una vez más, su utilidad en el aula de lenguas y culturas extranjeras como un vector de conocimiento. $\mathrm{El}$ docente debe tener claro que se requiere de un enfoque transversal que integre el lenguaje auténtico del libro y los aspectos socioculturales, junto 
con las competencias en producción y recepción oral y escritas, para que el estudiante complemente el desarrollo de las diferentes competencias que produzca en ILE o en la lengua extranjera de su interés, por medio de la comunicación.

\section{Las aventuras de Huckleberry Finn: un instrumento literario para entender la cultura y la sociedad del siglo XIX en los Estados Unidos}

Como complemento a la parte didáctica en la implementación del texto, hay otro tipo de aporte de la obra de Twain para el aula de ILE y culturas extranjeras; este está ligado a una serie de elementos narrativos e históricos que contribuirían al entendimiento de la cultura, la sociedad y sus problemáticas, que son plasmados por el autor.

En primer lugar, vemos como la literatura por medio de la lengua facilita la herencia de saberes y costumbres. Según Veblen (1974, citado en Busby, Korstanje y Mansfield, 2011, p. 22), un idioma es un prerrequisito del ser humano para la socialización y la narración de eventos, con el fin vincularse a un grupo cultural. De igual manera, Bram (1961, citado en Busby, Korstanje y Mansfield, 2011, p. 23) asegura que el lenguaje forma parte de la herencia del individuo y de la historia; así mismo, es el vehículo por el cual la herencia cultural es expresada y concebida. Por lo tanto, cada cultura ha evolucionado de maneras diferentes, cada cultura tiene su propio modo de expresión. En efecto, la obra de Twain muestra cómo fue su infancia en las orillas del río Mississippi. Esto se evidencia en el sinnúmero de eventos graciosos, bizarros y aventureros que tuvieron lugar cuando el joven Huck (Huckleberry) exploró en compañía de amigos y desconocidos los estados y lugares que rodeaban el río Mississippi. Lo anterior, conlleva al análisis de ciertos temas que explicitan los contenidos narrativos e históricos de la obra de Twain, con el propósito de entender la cultura norteamericana en el siglo XIX y, por qué no, en la actualidad.

\section{La religión}

Dicen que la religión es el opio de pueblo. Al ser un tema tan delicado en materia de sensibilidades, es necesario entender por qué los Estados Unidos 
es un país altamente religioso, así no lo parezca. Actualmente, el presidente Donald Trump cuenta con el respaldo de importantes líderes religiosos norteamericanos que ven con beneplácito ciertas decisiones políticas controversiales y de tinte religioso planteadas por el mandatario. Por ejemplo, la decisión de reconocer a Jerusalén como la capital del Estado de Israel fue celebrada no solamente por el mundo judío, sino también por la comunidad cristiana internacional. Eso sin profundizar en el papel que tiene su vicepresidente, Mike Pence, político conservador con una fuerte influencia cristiana, quien está de acuerdo con políticas como la prohibición del aborto y se opone al matrimonio igualitario y la legalización de las drogas, entre otras. Esta realidad contemporánea tiene sus bases en el pasado; por ello, es importante examinar lo que dice la obra literaria de Twain, para vincular la historia con el presente.

En Las aventuras de Huckleberry Finn se puede identificar a dos personajes cuyas herencias culturales y religiosas formaron parte de la idiosincrasia norteamericana del siglo XIX. Uno de estos personajes es Miss Watson: una mujer adulta, soltera y con fuertes convicciones religiosas, las cuales intenta imponer a Huck a través de amenazas. Así, vemos al inicio del libro cómo intenta corregir la conducta del niño mediante la imposición de miedos: "Then, when she told me about hell and said that it was where the bad people go when they die, I said that I wished that I was there already" (Twain, 1885, p. 2).

Twain pretendía mostrar cómo se infundía el miedo a los demás por medio de la instrumentación de principios religiosos como el cielo y el infierno, siendo el primero la recompensa y el segundo el castigo. De esta manera, si la persona hacía algo correcto, según la moral de la época, era premiada y enviada al cielo; de lo contrario, era condenada al infierno. Aunque esto no ha cambiado en la actualidad, contribuye a deducir el porqué del actuar de las personas en esa época o, incluso, hoy en día, lo cual podría interpretarse como un adoctrinamiento ideológico o un refuerzo moral y ético, para que la persona obre de acuerdo con unos parámetros socialmente aceptados, impuestos por una cultura dominante. Este es el caso de otro personaje importante, Jim: él es un esclavo negro que escapó con Huck. No sabía leer, ni escribir. En la época de la esclavitud, era eficaz mantener una fuerza de trabajo analfabeta que solo supiera obedecer órdenes; para lograrlo, el blanco, quien era el amo y señor no solo de tierras, sino de los esclavos, enseñaba, manipulaba y tergiversaba la palabra de Dios, con 
el fin de controlar las mentes y las conductas de estas personas. Una vez adoctrinados, los afroamericanos eran más dóciles de manejar.

Para ilustrar lo anterior, en una ocasión, Jim recordó una historia del rey Salomón que se encuentra en la Biblia. La comprendía perfectamente y, aunque no era un gran pensador, reflexionaba acerca de las acciones del rey:

The Bible says that King Solomon was the wisest man who ever lived, but he had many, many wives and I don't think that was so wise. Think of all the talking and the noise. And remember the time that two women came to him and each claimed to be the mother of the same baby. What does King Solomon say? He says that he'll cut the baby into two pieces and give each woman a piece. Now what can a woman do with half a baby? Do you think that was wise? (Twain, 1885, p. 47)

A pesar de su ingenuidad, ni Jim ni muchos esclavos veían la instrumentalización de la Biblia para los intereses oscuros del amo, que terminaba en un adoctrinamiento, porque era lo único que se permitía pensar y creer en ese entonces. A través del tiempo, el cristianismo se transformó en un modo de vida que se practica hoy en día. Los estadounidenses tienen fuertes raíces y creencias. A pesar de la persecución que se dio a las personas con distintas creencias religiosas, actualmente es un país que otorga el derecho de libertad de culto. El diario El Mundo, en su versión digital, ofrece las siguientes cifras: "Más de 100 millones de estadounidenses —un tercio de la población del país - están bautizados y, de ellos, en torno a 70 millones - las cifras varían según las encuestas - siguen definiéndose como parte del 'rebaño de Pedro”, (Pardo, 2015b). Esto demuestra la gran acogida de la tradición cristiana en el seno de la sociedad afroamericana.

Otro aspecto interesante de este análisis es observar cómo hoy en día los afroamericanos siguen con fervor las tradiciones cristianas que fueron heredadas desde sus antepasados a través de un proceso de aculturación, el cual viene desde la esclavitud en África. Un claro ejemplo de esto se refleja nuevamente en El Mundo: la Iglesia Metodista Episcopaliana Afroamericana de Emanuel (AME, según sus siglas en inglés) cuenta con más siete millones de miembros, todos de la comunidad afro. Esta iglesia es un referente fidedigno de lo que significó la esclavitud de los Estados Unidos, ya que existen fuertes vínculos históricos que rodean tal centro religioso: “[...] en pleno casco histórico de Charleston - la capital de Carolina del 
Sur, el primer estado en proclamar su independencia de Estados Unidos en 1861 para mantener la esclavitud-juega un papel central” (Pardo, 2015a). El otro hecho histórico que hay que resaltar es la iglesia fundada por "negros libertos":

[...] la iglesia de Emanuel es muy especial. Es el primer templo de la AME que se abrió fuera de la ciudad de Philadelphia, donde en 1787, Richard Allen y Absalon Jones-dos negros libertos-habían fundado el germen de esta organización religiosa: la Sociedad Negra Libre. Era un grupo con un fuerte sentido religioso, orientado a "nosotros, los africanos libres y sus descendientes", y que trataba de esquivar las restricciones a la asociación de personas negras vigente en la ciudad, en la que la esclavitud ya había sido abolida. (Pardo, 2015a)

De este modo, se evidencia que la tradición cristiana que fue enseñada a las comunidades negras del entonces sigue siendo muy influyente. Actualmente, es parte de su fe y su estilo de vida.

\section{La esclavitud}

Este es otro de los temas principales que sobresale en la obra de Twain (1885). Sin lugar a duda, es uno de los eventos trágicos más abominables de la historia. En Las aventuras de Huckleberry Finn, Twain describe una serie de eventos que revelan la tragedia de los esclavos afroamericanos. En consecuencia, se discernirá acerca de dos hechos clave del libro, con el fin de visualizar lo que aporta la obra literaria a la comprensión de esos hechos históricos, los cuales tienen cabida en la discusión en la clase de ILE y han repercutido en la vida actual, no solamente de las comunidades que fueron afectadas, sino como lección moral para que no se vuelvan a repetir.

Jim

A Jim, el esclavo de miss Watson, lo llaman Nigger Jim. Actualmente, la palabra nigger es un modismo inglés que denota de manera despectiva una persona de color. La personalidad de Jim muestra que es una persona ignorante (como muchos esclavos de ese entonces), ingenua y solidaria. Él cree en mitos que son originarios de sus antepasados, por ejemplo, existía la creencia en esa época de que los pelos de un asno tenían poderes, como el de permitir a la persona ver el futuro: "Jim, had a large hair ball that had 
been taken from the stomach of an ox. Jim thought that the hair ball had special powers. He said that it had the ability to tell what would happen in the future" (Twain, 1885, p. 10). De igual manera, al ser mordido por una serpiente, Jim piensa que cortar la cabeza y la piel del animal, para luego cocinarla y comerla, lo sanaría:

Jim told me to cut off the snake's head and throw it away. Then he told me to remove the snake's skin and cook a piece of its body. I did that, and Jim ate what I had cooked. He said that this would cure him. (Twain, 1885, p. 31)

Lo anterior implica reflexionar acerca de los conocimientos ancestrales que dominaban, especialmente cuando se trababa de medicina curativa y rituales.

Jim escapa de miss Watson para embarcar en una seria de aventuras con Huck. A pesar de ser bien tratado por ella, Jim huye porque iba a ser vendido y llevado a una gran colonia de negros esclavos que se encontraba en Nueva Orleans. Esa preocupación era común dentro de los esclavos: al igual que Jim, muchos tenían buenos dueños, pero al ser vendidos su destino era incierto, ya que no sabían a dónde iban a ir exactamente, ni quién los iba a comprar, ni para qué.

Adicionalmente, Jim, tal como otros esclavos que se atrevían a dejarlo todo, tenía que refugiarse en las peores condiciones, lo que hacía que su existencia fuera aún más penable que estar con sus dueños. Por ejemplo, al escapar con Huck, ambos estuvieron expuestos a enfermedades y peligros, como el de la serpiente o como cuando llovió tan fuerte que la balsa en la que iban se hundió y casi mueren ahogados por las fuertes corrientes del río Mississippi. La mayoría de los esclavos tenían que estar escondidos, porque en esa época eran cazados y torturados por tales actos de rebeldía.

\section{Los esclavos en la familia Grangerford}

Tener un esclavo en el siglo XIX era un lujo. Solo los grandes terratenientes y gente adinerada ostentaban ante la sociedad mostrando sus sirvientes. La familia Grangerford de "blancos”, bastante popular y ricos. Poseían grandes terrenos con ganado y cultivos. Era tanto el poder que cada integrante poseía un esclavo, cuando Huck fue acogido por ellos, también le fue dado un esclavo, llamado Buck: "Each member of the family had a black slave to work for him, I was given a black slave, too” (Twain, 1885, p. 61). 
Lamentablemente, esta situación posicionaba a las familias de ese entonces en un círculo social aristocrático, el cual mostraba poco o nada de respeto por las comunidades afro. Sin embargo, resulta fascinante que, a pesar de las fuertes diferencias raciales y económicas, se formaban ciertos vínculos entre amo y esclavo. Miss Sophia y Charlotte, hijas del coronel Grangerford, desde niñas tuvieron de cerca a sus esclavos, quienes las vieron crecer y volverse mujeres. En muchas ocasiones, los esclavos también formaban parte de los conflictos familiares: este es el caso de la riña que había entre las familias Grangerford y Shepherson. En un diálogo, Buck le dice a Huck que quiere dispararle a Harney, miembro de los Shephersons:

As soon as I was alone with Buck, I asked him:

—Did you want to shoot Harney?

-I certainly did.

- Has Harney ever attempted to harm you?

-No. He's never hurt me.

—Then why did you want to kill him?

-Because of the feud. (Twain, 1885, pp. 62-63)

Pese a que eran esclavos, había un vínculo de pertenencia y de apoyo con sus amos blancos. Finalmente, los sirvientes estaban mejor enterados que los dueños de casa: aunque su presencia era ignorada, ellos sabían todo lo que acontecía, unas veces por ser testigos, otras veces por ser los oídos de sus amos. Cuando miss Sophia escapó con su amado, quien era de la familia Shepherson, Buck y los demás sirvientes ya sabían de lo ocurrido, entonces él le contó a Huck:

—Don't you know?

-No, I don't know anything.

- Miss Sophia ran off in the night to get married to Harney Shepherdson. When the family learned about it a short while ago, they all rode off on their horses carrying guns. (Twain, 1885, p. 65)

En este orden de ideas, se puede observar cómo Twain (1885) muestra el lado más humano del esclavo negro: él quiere dar a entender que es un ser que piensa, bondadoso y fiel, mucho más de lo que puede ser un blanco, contrario a lo que se pensaba en aquel entonces: 
Certainly, one of Twain's goals was proving to the entire universe that a slave too, was a man, that blacks were not different from whites since the latter too were subjects to the same follies as blacks. Besides, in some cases, Mark Twain presented a black as being kinder, more loyal and more moral than whites. (Nyirubugara, 2001).

\section{Apreciaciones didácticas de los elementos culturales transmitidos por el texto de Twain}

En el análisis didáctico que se hizo anteriormente, el texto Las aventuras de Huckleberry Finn esbozaría exponencialmente una serie de elementos tantos lingüísticos como culturales de los Estados Unidos, los cuales son de gran utilidad para la enseñanza a estudiantes de licenciatura en ILE.

Al partir de la noción de infundir en los estudiantes la construcción del sentido, es clave como docentes hacer lecturas dirigidas o "metódicas", con el propósito de que los fenómenos de religión y esclavitud del siglo XIX en Norteamérica puedan ser entendidos fácilmente por los estudiantes de ILE. En este sentido, dos enfoques serían claves y podrían trabajarse de manera conjunta: el de Vigostky (2010) — el ZPD — y el de Descotes, Jordy y Langlade (1993):

La lecture méthodique met au contraire l'accent sur le processus de constructions de sens. Dans le cadre de la clase, elle opère un déplacement qui va, grossièrement, du sensexpliqué par l'enseignant aux élèves au sens produit par les élèves avec l'aide de l'enseignant.' (p. 29)

Así, lo que se sugiere es hacer una actividad de prelectura, que incluya una investigación de la religión y de la esclavitud durante el siglo XIX en Estados Unidos. Luego, se discutirán las lecturas para contextualizar estos temas y se dará inicio a una lectura dirigida del texto de Twain con los estudiantes en clase; en esta, se deben discutir los temas ya mencionados, con el fin de dar solución al problema de comprensión lectora de un texto que, debido a su origen extranjero, puede crear antagonismos en el modo

"La lectura dirigida se enfoca en el proceso de la construcción del sentido. En el marco de la clase, ella efectúa un desplazamiento que parte del sentido explicado por el docente a los alumnos, al sentido producido por los alumnos con la ayuda del docente". 
de construir el sentido y la interpretación de ideas. Dado que no todos los estudiantes piensan igual o saben las mismas cosas, se necesita la explicación por parte del docente de ciertos símbolos y rasgos culturales que este, como vector del idioma y al brindar su experiencia con la sociedad que lo habla, puede compartir a través de la interacción con el individuo que no ha tenido ningún tipo de acercamiento con un texto escrito por un autor norteamericano y que no conozca muy bien de qué manera influyó la religión y la esclavitud en la modernidad. Este trabajo colaborativo contribuye a minimizar el riesgo de creación de estereotipos en las aulas de clases e incluso estigmatizaciones, en este caso, hacia las personas afro: "Many students have simplistic understandings of culture and cultural identity, which leads to essentialized expectations of human behavior as people reduce the complexity of what it means to be human to labels and stereotypes" (Schall, s. f.)

De ahí que se requiere de un trabajo colaborativo en el aula de ILE y lenguas extranjeras, para que aquellas representaciones culturales generalizadas y subjetivas pasen a un plano objetivo y, así, el estudiante por medio del texto tome la iniciativa de reflexionar, por ejemplo, por qué en 114 Colombia se defiende tanto a las comunidades afro e indígenas. Esto solo es posible si desde el dispositivo didáctico creado para la enseñanza del inglés a través de Las aventuras de Huckleberry Finn se conecta con las realidades del estudiante de ILE.

Lo que ocurre en la obra de Twain (1885) está basado en hechos comprobables, fragmentos de un capítulo oscuro del ser humano. Esto hace que se deba tener un referente en el estudio del libro; incluso, aportaría a la sensibilización del estudiante con respecto a la importancia de resaltar el respeto a la diversidad de razas y religiones. El estudiante se convertirá en un actor social integro, receptivo y respetuoso con sus semejantes al haber sido instruido de este modo por su docente. Adicionalmente, sería valioso que como estudiante de lenguas y culturas extranjeras aprecie el aporte cultural que personas como los afros han dejado en nuestra sociedad. Por ejemplo, se podría mostrarle al estudiante de licenciatura en ILE o de lenguas extranjeras, durante uno de esos ejercicios de lecturas guiadas, cómo la esclavitud influyó fuertemente sobre muchos de los líderes afros norteamericanos e incluso colombianos. Para ilustrar lo anterior, se citará a un histórico político afronorteamericano: Martin Luther King, quien, en su famoso discurso conocido como I Have a Dream (1963), cuestiona la Norteamérica racista y clasista en la que tener un color de piel diferente al 
blanco puede considerarse un inconveniente: "I have a dream that my four little children will one day live in a nation where they will not be judged by the color of their skin but by the content of their character" (Luther King, s. f.). Por eso, la noción del referente es necesaria para relacionar un texto con el mundo real: "Si le monde des romans, des pièces de théâtre, des poèmes n'est bien entendu pas sans rapports avec le monde réel, il ne peut pas pour autant être confundu avec lui, ni lui être réduit”3 (Descotes, Jordy y Langlade, 1993, p. 41).

De esta manera, Twain (1885) contribuiría con dos elementos clave en el aula de clase: lo literario, para el aprendizaje de lenguas extranjeras, y lo cultural, para la formación personal del individuo.

\section{Conclusión}

La literatura suele observarse, por lo general, como un instrumento para desarrollar la competencia de la comprensión escrita. No obstante, esta herramienta tiene cualidades lingüísticas y culturales extraordinarias que permiten una enseñanza transversal e integral; entre ellas se encuentran las competencias lingüísticas, cognitivas, literarias y culturales. El diseño de un dispositivo didáctico que le permita al estudiante de licenciatura en ILE, o a cualquier otro estudiante de idioma extranjero, ser el vector de conocimientos es indispensable. Este tiene que ser concebido según las necesidades de aprendizaje y comunicativas del individuo. Por esta razón, en esta investigación se escogió el libro Las aventuras de Huckleberry Finn, de Mark Twain, el cual se puede estudiar en un establecimiento de educación superior con público de nivel intermedio A2+ y B1.

Por otra parte, el aporte cultural de la obra conlleva a una reflexión acerca de dos temas fundamentales que han afectado la humanidad, ya sea positiva o negativamente: la religión y la esclavitud. Al respecto, la reflexión y el debate de estas cuestiones provocarían un razonar distinto en la vida profesional y personal del estudiante universitario, ya que rompería con los falsos estereotipos y estigmas que tiene acerca de ellos. Esto se vería reflejado en la calidad de su formación y, por supuesto, en su actividad docente:

"Si el mundo de las novelas, de las obras de teatro, de los poemas, no tiene relación con el mundo real, no puede ser por ende confundido con él, ni lo pueden minimizar". 
sería un ente analítico, objetivo y respetuoso, que puede hablar con hechos ante una situación que esté ligada con la religión o la esclavitud.

Por lo tanto, la literatura no solo forja intelectos, también moldea y transforma las vidas y los mundos de las personas: "[ $[.$.$] a whole new world$ had begun to take shape - a world that, like most inner literary landscapes, is all the more enticing for being self-fashioned and, as such, a fundamental part of the experience that makes us who we are" (Taylor, 2016).

\section{Referencias}

Busby, G., Korstanje, M. y Mansfield, C. (2011). Madrid: literary fiction and the imaginary urban destination. Journal of Tourism Consumption and Practice, 3(2), 20-37. Recuperado de https://www.researchgate.net/publication/264250444_ Madrid_Literary_Fiction_and_the_Imaginary_Urban_Destination

Consejo de Europa. (2002). Marco común europeo de referencia para las lenguas: aprendizaje, enseñanza, evaluación. Madrid: Instituto Cervantes, Ministerio de Educación y Grupo Anaya.

Chauvet, A. (2008). Référentiel de programmes pour l'Alliance Française, élaboré à partir du Cadre Européen commun: A1, A2, B1, B2, C1, C2. París: Ediciones CLE International.

Gómez Rodríguez, L. F. (2015). La influencia del texto literario en el aprendizaje del inglés como lengua extranjera: de la teoría a la práctica. Forma y Función, 28(2), 83-109.

Descotes, M., Jordy, J. y Langlade, G. (1993). Le projet pédagogique en Français. París: Ediciones Bertrand-Lacoste.

Federación de Enseñanza de Comisiones Obreras de Andalucía. (2011). La importancia de la literatura en la enseñanza-aprendizaje de una lengua extranjera. Temas para la Educación: Revista Digital para Profesionales de la Enseñanza, (13). Recuperado de https://www.feandalucia.ccoo.es/docu/p5sd8298.pdf

Luther King, M. Jr. (s. f.). I have a dream, realizado el 28 de agosto de 1963 en el Lincoln Memorial, Washington D. C. Recuperado de http://www.americanrhetoric.com/speeches/mlkihaveadream.htm

Mendoza Fillola, A. (2008). La utilización de los materiales literarios en la enseñanza de lenguas extranjeras. Alicante: Biblioteca Virtual Miguel de Cervantes. Recuperado de http://www.cervantesvirtual.com/obra/la-utilizacion-de-materialesliterarios-en-la-ensenanza-de-lenguas-extranjeras--o/ 
Nyirubugara, O. (2001). Mark Twain's Satirical Approach to mid-19th Century American Society as Depicted in The Adventures of Huckleberry Finn (tesis de grado). Universidad de Bangui, Bangui, República Centroafricana. Recuperado de http://www. olny.nl/Research_Recherche/Memoires_Theses/Nyirubugara_Twain.pdf

Papo, E. y Bourgain, D. (1989). Litterature et communication en classe de langue. París: Didier.

Pardo, P. (2015a). Una iglesia emblemática para los negros de EEUU. El Mundo, 18 de junio. Recuperado de http://www.elmundo.es/internacional/2015/06/18/5 5826235e2704e50348b456d.html

Pardo, P. (2015b). Estados Unidos, un país (en el fondo) muy católico (a su manera). El Mundo, 24 de septiembre. Recuperado de http://www.elmundo.es/internaci onal/2015/09/24/5602d30346163f706a8b4596.html

Pérez Porto, J. y Merino, M. (2008). Definición de literatura. Definición.de. Recuperado de https://definicion.de/literatura/

Real Academia Española. (2018). Diccionario de la lengua española. Recuperado de https://dle.rae.es/?id=NR70JFl

Schall, J. M. (s. f.). Exploring cultural identity through literature. World of words. Recuperado de https://wowlit.org/on-line-publications/stories/storiesiv6/6/

Taylor, D. J. (2016). How the books we read shape our lives. The Independent, 3 de enero. Recuperado de https://www.independent.co.uk/arts-entertainment/books/features/how-the-books-we-read-shape-our-lives-a6794581.html

Twain, M. (1885). Las aventuras de Huckleberry Finn. Londres: Chatto \& Windus.

Vigotsky, L. (2010). Pensamiento y lenguaje. Madrid: Paidós.

Villalobos, J. (2017). Uso del diario interactivo para fomentar la escritura en ELE: volviendo al papel y al lápiz. Matices en Lenguas Extranjeras, (11), 45-75. Recuperado de https://revistas.unal.edu.co/index.php/male/article/view/71854/65837 